\title{
TEORES DE COMPOSTOS ANTIOXIDANTES ENTRE TIPOS DIFERENTES DE ARROZ ${ }^{1}$ \\ CONTENTS OF ANTIOXIDANT COMPOUNDS AMONG DIFFERENT TYPES OF RICE
}

\author{
Ludmila Gontijo Pacheco², Luciene Alves ${ }^{3}$, Flávia Della Lúcia ${ }^{4}$ Olga Luisa Tavano ${ }^{5}$
}

\section{RESUMO}

O presente estudo tem como objetivo comparar os teores de compostos fenólicos totais, flavonoides e atividade antioxidante de diferentes tipos de arroz disponíveis no comércio de Uberaba-MG, com e sem processamento térmico. Foi possível a obtenção de 18 amostras de arroz, de distintas marcas. Todas as amostras apresentaram resultados para fenólicos totais e flavonoides, mesmo aquelas de grãos brancos e polidos, porém grãos com o pericarpo colorido apresentaram valores mais expressivos, o mesmo ocorrendo para seus potenciais antioxidantes. Todas as amostras apresentaram redução nos resultados de fenólicos totais e flavonoides após o cozimento, com exceção de uma amostra de arroz integral (Marca 2). As amostras orgânicas de arroz polido e arroz integral apresentaram teores de fenólicos totais e flavonoides superiores às seus correspondentes não orgânicos. O mesmo pode ser observado para os dados referentes ao potencial antioxidante destas amostras, com exceção da amostra de arroz polido em sua forma sem cozimento. Observou-se que as amostras de arroz de genótipos pigmentados apresentam altos teores de fenólicos totais, entre eles os flavonoides, o que poderia explicar seus altos valores para atividade antioxidante. As amostras de grãos integrais e orgânicos também se destacaram. Estes dados indicam que a substituição do arroz branco por grãos integrais, e, especialmente por grãos pigmentados, pode ser benéfica, contribuindo para o aumento da ingestão de componentes antioxidantes.

Palavras-chaves: Compostos Fenólicos, DPPH, Flavonoides, Radicais Livres.

\section{ABSTRACT}

The study aims to compare the levels of total phenolic compounds, flavonoids and antioxidant activity of different types of rice available in the Uberaba-MG trade, with and without thermal processing. It was possible to obtain 18 samples of rice from different brands. All samples presented results for total phenolic and flavonoid compounds, even those of white and polished grains, but grains with the colored pericarp presented more expressive values, the same occurring for their potential antioxidants. All samples showed reduced results in total phenolic and flavonoid after cooking, with the exception of brown rice sample (Mark 2). The organic samples of polished rice and brown rice showed higher levels of total phenolic and flavonoid than their non-organic counterparts. The same can be observed for data concerning the antioxidant potential of these samples, except for the sample of polished rice in its uncooked form. It was observed that the samples

${ }^{1}$ Trabalho de Conclusão de Curso.

${ }^{2}$ Graduada em Nutrição - Departamento de Nutrição - Instituto de Ciências da Saúde - Universidade Federal do Triângulo Mineiro - Uberaba - Minas Gerais - Brasil. E-mail: ludmilagontijo@hotmail.com

${ }^{3}$ Orientadora - Departamento de Nutrição - Instituto de Ciências da Saúde - Universidade Federal do Triângulo Mineiro Uberaba - Minas Gerais - Brasil. E-mail: lucienealves159@gmail.com

${ }^{4}$ Colaboradora - Faculdade de Nutrição - Universidade Federal de Alfenas - UNIFAL - Alfenas- Minas Gerais - Brasil. E-mail: dellaluciaf@gmail.com

${ }^{5}$ Orientadora - Faculdade de Nutrição - Universidade Federal de Alfenas - UNIFAL - Alfenas- Minas Gerais - Brasil. E-mail: tavanool@yahoo.com.br 
of pigmented rice genotypes have high levels of total phenolic, including flavonoids, which could explain their high values for antioxidant activity. Samples of whole grains and organic grains also stood out. These data indicate that replacing white rice with whole grains, and especially with pigmented grains, can be beneficial, contributing to the increased intake of antioxidant components.

Keywords: DPPH, Flavonoids, Free Radicals, Phenolic Compounds.

\section{INTRODUÇÃO}

O arroz (Oryza sativa L.) é um dos alimentos mais importantes da alimentação humana. Segundo dados da FAO (Food and Agriculture Organization of the United Nations) referentes a 2013, o consumo mundial médio de arroz foi de $54 \mathrm{~kg} / \mathrm{per}$ capita/ano, sendo este o responsável por cerca de $23 \%$ da ingestão calórica total, ou seja, é a maior fonte calórica entre os alimentos registrados, além de colaborar com 10,13 gramas de proteínas per capita/ por dia (a terceira maior fonte proteica, atrás somente do trigo e da carne). Para a América Latina, os dados da FAO (2013) indicam que o arroz colabora com uma média de 5,8 gramas de proteínas per capita/dia e $291 \mathrm{kcal}$ per capita/dia, com um consumo anual total de $28,72 \mathrm{~kg}$ per capita. Já para o Brasil os dados são um pouco mais altos, sendo consumidos cerca de $32 \mathrm{~kg}$ per capita/ano.

Barata (2005) apresenta em sua pesquisa a preferência dos consumidores com relação ao tipo de arroz consumido na Região Metropolitana de Porto Alegre (2004), onde cerca de 75\% dos consumidores declararam consumir o arroz polido tipo $1,5 \%$ o tipo 2 , cerca de $18 \%$ o arroz parboilizado e 4\% o arroz integral. Menos de 1\% afirmava consumir outros tipos de arroz, citados apenas entre os consumidores de maior renda familiar. Estes dados são semelhantes aos divulgados pela Empresa Brasileira de Pesquisa Agropecuária (EMBRAPA, 2005) com relação ao panorama nacional, em que pesquisas de mercado apontam para uma migração de consumo do arroz branco polido Tipo 2 para o Tipo 1 e o Parboilizado. Desta forma, segundo a EMBRAPA, o arroz Tipo 1 representa hoje 70 a $80 \%$ do mercado brasileiro de arroz polido branco (a classificação do arroz está baseada no estado físico dos grãos, que varia em relação a quebra dos mesmos, de 1 a 5, e ao tamanho de grão, curto ou longo), e ainda, o arroz Parboilizado, encontra-se em expansão, representando 20\% (1,5 milhões de toneladas) da demanda de arroz beneficiado, que é de 7,2 milhões de toneladas/ano.

Embora o arroz faça parte dos hábitos alimentares dos brasileiros há cerca de um século (NAVES, 2007), como dito anteriormente, este é consumido basicamente na forma de grãos brancos e polidos, sendo os outros tipos ainda pouco consumidos, em parte por seu elevado custo e também pela falta de hábito, já que não faz parte da cultura do brasileiro o consumo de arroz pigmentado e mesmo o integral, embora existam diversas variedades de arroz disponíveis no mercado, variáveis em relação à pigmentação que está presente no pericarpo, resultando, por exemplo, no arroz pigmentado vermelho, preto ou castanho e que podem ser encontrados em suas formas integrais ou parboilizados 
(JUN et al., 2012). Embora o arroz seja considerado essencialmente como fonte de carboidratos, pode fornecer outros nutrientes como proteínas, fibras, minerais e vitaminas, especialmente se consumido em suas formas integrais. $\mathrm{O}$ arroz colhido passa por processos que o transformam na forma adequada para consumo humano. O processo inicia-se com a remoção da casca não comestível, surgindo assim o grão ou arroz integral. O endosperma do arroz, também conhecido como arroz polido ou arroz branco, é produzido por um processo de polimento adicional que retira a camada de farelo de todo o grão. Além dos nutrientes, diferentes pesquisas demonstram que o arroz pode ser fonte de outros componentes bioativos, como compostos fenólicos, cujas concentrações nos grãos apresentam uma relação positiva com a maior coloração do pericarpo. Em geral, o endosperma do arroz é o preferido devido às suas propriedades sensoriais desejáveis e estabilidade de armazenamento (GOUFO; TRINDADE, 2014), mas, muitos compostos antioxidantes, que estão presentes principalmente no pericarpo, podem ser removidos durante o processamento dos grãos (Walter et al., 2007). A composição e concentração de fenólicos podem variar de acordo com coloração, polimento ou tratamento térmico sofrido (VICHAPONG, 2010). Pesquisas, como a realizada por Walter et al. (2013), demonstram que os genótipos de colorações preta, avermelhadas e marrons apresentam alto teor de compostos fenóli$\cos$, e relacionam estes teores às suas maiores capacidades como potenciais fontes de antioxidantes.

Considerando que uma substância antioxidante pode ser definida como aquela que significativamente diminui ou inibe a oxidação de um determinado substrato, os antioxidantes podem ser classificados como os que apresentam atividade enzimática ou aqueles sem atividade enzimática. Na segunda classe, estão moléculas que vão interagir com as espécies radicalares e "sacrificar" sua própria integridade molecular para evitar alterações no meio celular; nesta classificação incluem-se os antioxidantes naturais e sintéticos (ANGELO e JORGE, 2007; PEREIRA, VIDAL e CONSTANT, 2009). Podemos distinguir diferentes mecanismos de ação para tal atividade, como a ação de captura de radicais livres ou de reparação da oxidação sofrida pelo substrato (NIKI, 2010).

O estresse oxidativo vem sendo associado a várias patogenias e desordens metabólicas, impulsionando pesquisas que buscam esclarecer as funções e aplicações de compostos antioxidantes na manutenção e promoção da saúde humana (DEMMING-ADAMS e ADAMS, 2002). Vários componentes dos alimentos vêm sendo estudados por apresentarem função antioxidante, tais como o ácido ascórbico, o $\beta$-caroteno, o $\alpha$-tocoferol, o zinco, o selênio e os compostos fenólicos, cada vez mais alvo de estudos (ZIMMERMANN e KIRSTEN, 2008). Os compostos fenólicos constituem um dos mais numerosos grupos de metabólitos secundários em plantas, com mais de 8000 estruturas fenólicas conhecidas; sendo essenciais para a proteção das plantas contra herbívoros e microorganismos patogênicos (ANGELO e JORGE, 2007; SILVA et al., 2010; SOOBRATTEE et al., 2005). Entre os fenóis existentes, se destacam os ácidos fenólicos, os flavonoides, os fenóis simples, cumarinas, taninos, ligninas e tocoferóis. Os flavonoides, uma das categorias de compostos fenólicos mais associadas à atividade antioxidante, consistem de um esqueleto de 15 carbonos que estão organizados em dois 
anéis aromáticos interligados por uma cadeia de três carbonos. São reconhecidos tanto pela sua capacidade de doar elétrons, quanto de parar reações em cadeia (HUDSON et al., 2000; CHO et al., 2013).

Dados que demonstrem a vantagem de consumo de grãos de arroz branco em sua forma integral ou mesmo de outros tipos de grãos de arroz mais pigmentados em comparação ao arroz polido, podem colaborar na discussão das vantagens da incorporação destas formas de consumo dos grãos na dieta da população. O presente estudo tem como objetivo comparar os teores de compostos fenólicos totais, flavonoides e atividade antioxidante de diferentes tipos de arroz disponíveis no comércio de Uberaba-MG, antes e após seu processamento térmico.

\section{MÉTODOS}

\section{Amostras}

Em consulta aos mercados de comercialização de produtos alimentícios do município de Uberaba-MG, observou-se a disponibilidade de 18 diferentes amostras de arroz, de distintas marcas, e todas disponíveis em embalagens fechadas, cujas principais características são apresentadas na Tabela 1. As amostras foram adquiridas, manualmente limpas retirando-se sujidades ou grãos partidos, e então parte destas foi triturada até granulometria de 60 mesh, correspondendo às amostras sem cozimento destinadas às análises. Outra parte das amostras foi submetida ao processamento térmico, simulando-se processo de cozimento com tempo de processamento e proporção massa de amostra: volume de água destilada estabelecidos de acordo com o recomendado nas embalagens das mesmas. Em seguida, as amostras foram imediatamente resfriadas e então congeladas para posteriormente serem liofilizadas e também trituradas até granulometria de 60 mesh.

Tabela 1 - Características das diferentes amostras de arroz obtidas no comércio local de Uberaba-MG.

\begin{tabular}{|lcc}
\hline \multicolumn{1}{c}{ Amostra } & Tipo & Classe \\
\hline Arbóreo Marca 3 & NC & NC \\
\hline Carnaroli Polido Marca 3 & 1 & Longo \\
\hline Momiji Polido Marca 7 & 1 & Curto \\
\hline Basmati Marca 3 & NC & longo fino \\
\hline Branco Agulinha Polido Marca 2 & 1 & longo fino \\
\hline Branco Polido Marca 2 & 1 & longo fino \\
\hline Orgânico Polido Marca 8 & 1 & longo fino \\
\hline Parboilizado Polido Marca 10 & 1 & longo fino \\
\hline Parboilizado Integral Marca 1 & 1 & Longo \\
\hline Parboilizado Integral Marca 3 & 1 & NC \\
\hline Integral Marca 2 & 1 & longo fino \\
\hline Integral Marca 5 & 1 & longo fino \\
\hline Integral Orgânico Marca 2 & 1 & longo fino \\
\hline Integral Orgânico Marca 9 & 1 & longo fino \\
\hline
\end{tabular}




\begin{tabular}{lcc} 
Castanho Integral Marca 6 & 1 & Médio \\
Preto Integral Marca 4 & 1 & Médio \\
Vermelho Marca 4 & 1 & Médio \\
Selvagem Marca 3 & NC & NC \\
\hline \multicolumn{2}{l}{ NC = não classificado } &
\end{tabular}

\section{Determinação de Umidade}

Para melhor efeito de comparação e expressão dos dados os resultados foram também expressos em base seca. Para tanto as amostras in natura de arroz tiveram suas determinações de umidade realizadas em estufa com circulação de ar, a $105^{\circ} \mathrm{C}$, até peso constante (HOROWITZ, 2000). Para as alíquotas submetidas ao processamento térmico o processo de liofilização a que foram submetidas foi utilizado para estimativa de seus teores de umidade/sólidos totais.

\section{Preparo dos extratos}

Para diferentes determinações, um extrato etanólico foi preparado, utilizando-se as amostras trituradas. As extrações foram realizadas com uso de solvente etanol 80\%, na proporção 1:40 (m/v), sob agitação constante $\left(1 \mathrm{~h} / 4^{\circ} \mathrm{C}\right)$, mantida sob proteção da luz, com posterior centrifugação $(7.000 \mathrm{rpm} /$ $5 \mathrm{~min} / 5^{\circ} \mathrm{C}$ ), como descrito por PEREIRA e TAVANO (2014). Os sobrenadantes foram armazenados sob refrigeração, em frascos de vidro envoltos em papel de alumínio até o momento das análises.

\section{Determinação da capacidade antioxidante pela captura de radical livre}

Para a determinação da capacidade antioxidante das amostras, utilizou-se método descrito por BRAND-WILLIAMS, CURVELIER e BERSET (1995), e modificado por PEREIRA e TAVANO (2014). Brevemente: $250 \mu \mathrm{L}$ dos extratos (obtidos como descrito em 2.3) foram adicionados de $1 \mathrm{ml}$ de solução de DPPH (2,2-diphenyl-1-picryl-hidrazil) 0,06 mM, em etanol 80\%. As absorvâncias foram monitoradas a $517 \mathrm{~nm}$ até estabilização da queda de absorção. Uma curva de calibração de DPPH foi previamente construída para utilização nos cálculos e os resultados expressos em $\mu \mathrm{Mols}$ de DPPH inibidos/g de amostra.

\section{Determinação de fenólicos totais}

Os teores de fenólicos totais solúveis nos extratos, foram determinados como descrito por CANO et al. (2003) e modificado por THOMAS et. al. (2010) utilizando-se reagente de Folin-Ciocalteu e curva de ácido gálico como padrão. Os resultados foram expressos em equivalentes de ácido gálico/g 
de amostra. O Índice Antioxidante de Fenóis (PAOXI) foi calculado como descrito por PEREIRA e TAVANO (2014) dividindo-se a atividade antioxidante da amostra ( $\mu$ mol de DPPH inibidos/g) pelo total de fenólicos da mesma amostra (mg/g).

\section{Determinação de flavonoides}

Para a determinação dos teores de flavonoides das amostras, utilizou-se método descrito por BOATENG et al. (2008), com algumas modificações. Brevemente: Em $250 \mu$ l do extrato etanólico, foram adicionados $1,0 \mathrm{ml}$ de água destilada, $75 \mu \mathrm{l}$ de solução de $\mathrm{NaNO}_{2}(5 \%)$. Após agitação e 5 minutos de incubação em temperatura ambiente foram adicionados $75 \mu 1$ de $\mathrm{AlCl}_{3}(10 \%)$, e após mais 5 min 0,5 $\mathrm{ml}$ de $\mathrm{NaOH} 1 \mathrm{M}$. As leituras foram realizadas a $510 \mathrm{~nm}$ e uma curva padrão de catequinas utilizada para os cálculos. Os resultados foram expressos em mg de equivalentes de catequinas/g de amostra.

\section{Análises estatísticas}

Todos os ensaios foram realizados em triplicada e os resultados foram expressos como médias. Utilizou-se a análise de variância (ANOVA) e teste de Tukey para comparação de médias com nível de significância de 5\% ( $\mathrm{p} \leq 0,05)$, com auxílio do programa Statsoft STATISTICA 8.0 (2007).

\section{RESULTADOS E DISCUSSÃO}

Para melhor efeito de comparação entre as amostras e mesmo com dados da literatura, todos os resultados foram expressos em base seca, ou seja, por grama de sólidos totais das amostras. Os teores de umidade das amostras antes e após cozimento são apresentados na Tabela 2 , indicando pouca variação entre as amostras. Os resultados dos teores de fenólicos totais e flavonoides das amostras com e sem cozimento foram expressos na Tabela 3.

Tabela 2 - Teores de umidade das diferentes amostras de arroz disponíveis no comércio local de Uberaba-MG, in natura e cozidas.

\begin{tabular}{lcc}
\hline \multirow{2}{*}{\multicolumn{1}{c}{ Amostras }} & \multicolumn{2}{c}{ Teores de Umidade (\%) } \\
\cline { 2 - 3 } & In natura & Cozida \\
\hline Arbóreo (Marca 3) & 10,36 & 72,08 \\
\hline Carnaroli Polido (Marca 3) & 9,63 & 64,46 \\
\hline Momiji Polido (Marca 7) & 11,75 & 66,20 \\
\hline Basmati (Marca 3) & 10,04 & 63,41 \\
\hline Branco agulhinha Polido (Marca 2) & 10,19 & 63,20 \\
\hline Branco Polido (Marca 1) & 9,98 & 65,93 \\
\hline Orgânico Polido (Marca 8) & 10,00 & 66,92 \\
\hline Parboilizado Polido (Marca 1) & 9,83 & 65,94
\end{tabular}




\begin{tabular}{|lcc|}
\hline Parboilizado Integral (Marca 3) & 10,30 & 59,50 \\
\hline Parboilizado Integral (Marca 1) & 8,77 & 62,18 \\
\hline Integral (Marca 2) & 9,55 & 45,40 \\
\hline Integral (Marca 5) & 9,88 & 55,20 \\
\hline Integral Orgânico (Marca 2) & 10,40 & 72,75 \\
\hline Integral Orgânico (Marca 9) & 10,56 & 49,62 \\
\hline Castanho Integral (Marca 6) & 9,23 & 58,80 \\
\hline Preto Integral (Marca 4) & 9,14 & 47,26 \\
\hline Vermelho Integral (Marca 4) & 10,06 & 50,26 \\
\hline Selvagem (Marca 3) & 7,91 & 39,47 \\
\hline
\end{tabular}

Todas as amostras apresentaram resultados para fenólicos totais e flavonoides, mesmo aquelas referentes aos grãos brancos e polidos, embora tenham sido observados nos grãos com o pericarpo colorido (preto, vermelho e castanho) valores bem mais expressivos em relação às outras amostras (Tabela 3), o mesmo ocorrendo para os teores de flavonoides. $\mathrm{O}$ arroz selvagem também apresentou valores de fenólicos totais e flavonoides superiores aos apresentados pelas amostras de arroz sem pigmentação, embora seus resultados tenham sido menores do que os genótipos de arroz coloridos. Todas as amostras apresentaram redução nos resultados de fenólicos totais e flavonoides após o cozimento, com exceção da amostra de arroz integral (Marca 2) que não apresentou redução significativa de seus valores de flavonoides (Tabela 3).

Tabela 3 - Conteúdo de compostos fenólicos totais e flavonoides em amostras de diferentes tipos de arroz disponíveis no comércio local de Uberaba-MG.

\begin{tabular}{|c|c|c|c|c|}
\hline \multirow[t]{2}{*}{ Amostras } & \multicolumn{2}{|c|}{$\begin{array}{c}\text { Fenólicos Totais } \\
\text { (mg EAG/g*) }\end{array}$} & \multicolumn{2}{|c|}{$\begin{array}{l}\text { Flavonóides } \\
\left(\mathrm{mg} \mathrm{EC} / \mathrm{g}^{*}\right)\end{array}$} \\
\hline & Crus & Cozidos & Crus & Cozidos \\
\hline Arbóreo (Marca 3) & $0,61^{\mathrm{jA}}$ & $0,09^{\mathrm{n} B}$ & $0,10^{\mathrm{n} A}$ & $0,05^{\mathrm{m} \mathrm{B}}$ \\
\hline Carnaroli Polido (Marca 3) & $0,53^{1 \mathrm{~A}}$ & $0,10^{\mathrm{m} \mathrm{B}}$ & $0,10^{\mathrm{n} \mathrm{A}}$ & $0,07^{\text {в }}$ \\
\hline Momiji Polido (Marca 7) & $0,74^{\mathrm{h} \mathrm{A}}$ & $0,22^{\mathrm{h} \mathrm{B}}$ & $0,12^{\mathrm{mnA}}$ & $0,06^{1 \mathrm{~B}}$ \\
\hline Basmati (Marca 3) & $0,45^{\mathrm{m} \mathrm{A}}$ & $0,21^{\text {i в }}$ & $0,062^{\mathrm{qA}}$ & $0,03^{\circ \mathrm{B}}$ \\
\hline Branco agulhinha Polido (Marca 2) & $0,21^{\mathrm{pA}}$ & $0,03^{\circ \mathrm{B}}$ & $0,09^{\circ \mathrm{A}}$ & $0,04^{\mathrm{n} \mathrm{B}}$ \\
\hline Branco Polido (Marca 1) & $0,40^{\mathrm{nA}}$ & $0,28^{\mathrm{g} \mathrm{B}}$ & $0,13^{1 \mathrm{~A}}$ & $0,05^{\mathrm{m} \mathrm{B}}$ \\
\hline Orgânico Polido (Marca 8) & $0,60^{\mathrm{jA}}$ & $0,12^{1 \mathrm{~B}}$ & $0,10^{\mathrm{n} A}$ & $0,08^{\text {B B }}$ \\
\hline Parboilizado Polido (Marca 1) & $0,26^{\mathrm{oA}}$ & $0,10^{\mathrm{n} \mathrm{B}}$ & $0,065^{\mathrm{pA}}$ & $0,05^{\mathrm{mB}}$ \\
\hline Parboilizado Integral (Marca 3) & $0,53^{1 \mathrm{~A}}$ & $0,37^{\mathrm{e}} \mathrm{B}$ & $0,23^{\mathrm{h} \mathrm{A}}$ & $0,18^{\mathrm{dB}}$ \\
\hline Parboilizado Integral (Marca 1) & $0,65^{\mathrm{iA}}$ & $0,19^{\text {ј в }}$ & $0,26^{\mathrm{fA}}$ & $0,17^{\mathrm{e} \mathrm{B}}$ \\
\hline Integral (Marca 2) & $0,58^{\mathrm{kA}}$ & $0,47^{\mathrm{d} \mathrm{B}}$ & $0,22^{\mathrm{i} A}$ & $0,22^{\mathrm{bcd} A}$ \\
\hline Integral (Marca 5) & $0,81^{\mathrm{gA}}$ & $0,30^{\mathrm{fB}}$ & $0,19^{\mathrm{j} A}$ & $0,09^{\text {h B }}$ \\
\hline Integral Orgânico (Marca 2) & $1,84^{\mathrm{fA}}$ & $1,12^{\mathrm{b} \mathrm{B}}$ & $0,25^{\mathrm{g} \mathrm{A}}$ & $0,12^{\mathrm{g} \mathrm{B}}$ \\
\hline Integral Orgânico (Marca 9) & $1,96^{\mathrm{e} A}$ & $1,09^{\mathrm{b} \mathrm{B}}$ & $0,32^{\mathrm{e} A}$ & $0,19^{\mathrm{d} B}$ \\
\hline Castanho Integral (Marca 6) & $15,32^{\mathrm{a} \mathrm{A}}$ & $0,29^{\mathrm{g}} \mathrm{B}$ & $1,18^{\mathrm{a} \mathrm{A}}$ & $0,22^{\mathrm{c} \mathrm{B}}$ \\
\hline Preto Integral (Marca 4) & $8,98^{\mathrm{cA}}$ & $0,80^{\mathrm{c} \mathrm{B}}$ & $0,60^{\mathrm{cA}}$ & $0,63^{\mathrm{a} \mathrm{B}}$ \\
\hline Vermelho Integral (Marca 4) & $11,75^{\mathrm{bA}}$ & $0,47^{\mathrm{dB}}$ & $1,05^{\mathrm{bA}}$ & $0,24^{\text {bc B }}$ \\
\hline Selvagem (Marca 3) & $3,13^{\mathrm{dA}}$ & $1,16^{\mathrm{aB}}$ & $0,40^{\mathrm{dA}}$ & $0,16^{\mathrm{fB}}$ \\
\hline
\end{tabular}

*Valores expressos como médias $(\mathrm{n}=3)$. Dados de uma mesma coluna ou linha seguidos pela mesma letra sobrescrita (minúsculas para colunas e maiúsculas para linhas de um mesmo ensaio) não apresentam diferença significativa entre si $(\mathrm{p}<0.05)$ pelo Teste de Tukey. Dados expressos como mg de equivalentes de ácido gálico (EAG) e equivalente de catequina (EC)/ g de amostra em base seca. 
É interessante destacar que as amostras orgânicas de arroz polido e arroz integral apresentaram teores de fenólicos totais e flavonoides superiores aos seus correspondentes não orgânicos, tanto em suas formas cruas quanto cozidas (Tabela 3). O mesmo pode ser observado para os dados referentes ao potencial antioxidante destas amostras, com exceção da amostra de arroz polido em sua forma sem cozimento (Tabela 4). De fato, o perfil de potencial antioxidante de todas as amostras foi semelhante ao observado para os teores de fenólicos totais, com as amostras coloridas apresentando os maiores valores de $\mu$ Mols de DPPH inibidos/g de arroz, tanto para amostras cruas como cozidas (Tabela 4). O cozimento também gerou redução significativa do potencial como antioxidantes de todas as amostras testadas.

Tabela 4 - Potencial antioxidante em amostras de diferentes tipos de arroz disponíveis no comércio local de Uberaba-MG.

\begin{tabular}{|c|c|c|c|c|}
\hline \multirow[t]{2}{*}{ Amostras } & \multicolumn{2}{|c|}{$\begin{array}{c}\text { Potencial antioxidante } \\
\left(\mu \text { Mols of DPPH inibidos } / g^{*}\right)\end{array}$} & \multicolumn{2}{|c|}{ PAOXI* } \\
\hline & Grãos Crus & Grãos Cozidos & Grãos Crus & Grãos Cozidos \\
\hline Arbóreo (Marca 3) & 1,39 ghi & ND & 2,29 & - \\
\hline Carnaroli Polido (Marca 3) & $1,50^{\text {ghi A }}$ & $0,86^{\mathrm{iB}}$ & 2,83 & 7,89 \\
\hline Momiji Polido (Marca 7) & $1,93^{\mathrm{bfg} \mathrm{A}}$ & $0,75^{\text {в в }}$ & 2,60 & 3,34 \\
\hline Basmati (Marca 3) & $1,34^{\text {ghi }}$ & ND & 2,98 & - \\
\hline Branco agulhinha Polido (Marca 2) & $1,74^{\mathrm{bc}}$ & ND & 8,28 & - \\
\hline Branco Polido (Marca 1) & $1,85^{\mathrm{bA}}$ & $0,19^{1 \mathrm{~B}}$ & 4,59 & 0,67 \\
\hline Orgânico Polido (Marca 8) & $1,54^{\text {ghi A }}$ & $0,85^{\text {i В }}$ & 2,54 & 6,80 \\
\hline Parboilizado Polido (Marca 1) & $0,51^{\mathrm{jA}}$ & $0,12^{\mathrm{mB}}$ & 1,98 & 1,18 \\
\hline Parboilizado Integral (Marca 3) & $2,06^{\mathrm{fA}}$ & $1,22^{\mathrm{h} \mathrm{B}}$ & 3,85 & 3,31 \\
\hline Parboilizado Integral (Marca 1) & $2,24^{\mathrm{fA}}$ & $1,98^{\mathrm{eB}}$ & 3,42 & 18,09 \\
\hline Integral (Marca 2) & $1,26^{\text {hi A }}$ & $0,26^{\mathrm{kB}}$ & 2,14 & 0,52 \\
\hline Integral (Marca 5) & $1,73^{\text {fghi A }}$ & $0,88^{\mathrm{i} \text { B }}$ & 2,12 & 2,89 \\
\hline Integral Orgânico (Marca 2) & $6,89^{\mathrm{e} A}$ & $1,60^{\mathrm{fB}}$ & 3,72 & 0,89 \\
\hline Integral Orgânico (Marca 9) & $6,81^{\mathrm{e} A}$ & $1,48^{\mathrm{gB}}$ & 3,47 & 1,35 \\
\hline Castanho Integral (Marca 6) & $41,06^{\mathrm{cA}}$ & $9,40^{\mathrm{b} \text { B }}$ & 2,68 & 100 \\
\hline Preto Integral (Marca 4) & $45,25^{\mathrm{bA}}$ & $26,94^{\mathrm{a} \mathrm{B}}$ & 5,03 & 33,54 \\
\hline Vermelho Integral (Marca 4) & $49,92^{\mathrm{a} \mathrm{A}}$ & $4,84^{\mathrm{c} \mathrm{B}}$ & 4,24 & 10,25 \\
\hline Selvagem (Marca 3) & $17,8^{\mathrm{dA}}$ & $3,15^{\mathrm{dB}}$ & 5,69 & 2,72 \\
\hline
\end{tabular}

*Valores expressos como média $(n=3)$, em base seca. Dados de uma mesma coluna ou linha seguidos pela mesma letra sobrescrita (minúsculas para colunas e maiúsculas para linhas) não apresentam diferença significativa entre si $(\mathrm{p}<0.05)$ pelo Teste de Tukey. ND = Não detectado.

**PAOXI = Índice Antioxidante de Fenóis, calculado dividindo-se a atividade antioxidante da amostra ( $\mu \mathrm{mol}$ de DPPH inibidos/g) pelo total de fenólicos da mesma amostra (mg/g).

De acordo com os resultados das Tabelas 3 e 4, não foi possível verificar uma clara influência do processo de parboilização sobre os parâmetros testados. Já no que se refere ao uso dos grãos integrais esta diferenciação parece ter influenciado positivamente os teores de compostos fenólicos dos grãos, uma vez que estes apresentaram em média resultados superiores à de seus correspondentes polidos (Tabela 3). Os percentuais de flavonoides em relação aos fenólicos totais variaram entre 13,67\% 
e 44,69\% para amostras de arroz branco, representando os menores percentuais entre as amostras de genótipos pigmentados: 6,73\% para amostra de arroz preto, 7,73\% para a amostra de arroz castanho e $8,99 \%$ para a amostra de arroz vermelho.

Quando foram calculadas as correlações entre o potencial antioxidante e o teor de fenólicos totais das amostras cruas, observou-se uma forte correlação positiva $\left(r=0,9494, r^{2}=0,9014\right.$, $p=0,000000$ ). Embora exista correlação entre os teores de fenólicos totais e os de flavonoides das amostras $\left(r=0,7359, r^{2}=0,5415, p=0,000499\right)$, e os flavonoides sejam considerados dentre os componentes fenólicos aqueles que mais representam compostos antioxidantes, quando os valores de inibição de DPPH foram correlacionados com os teores de flavonoides $\left(r=0,6864, r^{2}=0,4711, p=0,001657\right)$ apenas uma correlação moderada foi identificada.

Ainda que os flavonoides sejam considerados uma classe de fenólicos que apresenta maior capacidade como antioxidante, neste trabalho, embora tenha sido encontrada uma correlação entre os teores de flavonoides e o potencial antioxidante das amostras, quando os percentuais de flavonoides entre os componentes fenólicos totais foi observado, verificou-se que mesmo aquelas amostras com baixos percentuais de flavonoides apresentaram potencial antioxidante. De fato, aquelas amostras com baixos percentuais de flavonoides entre seus fenólicos totais, como as amostras de arroz preto, castanho e vermelho, apresentaram os maiores potenciais (Tabela 4). É sabido que a composição dos fenólicos dos vegetais é amplamente variável e que os diferentes compostos podem apresentar diferentes atividades como antioxidantes, o mesmo ocorrendo para os grãos de arroz (MIN, LUNG e CHEN, 2011). O Índice Antioxidante de Fenólicos (PAOXI) relaciona o teor de fenólicos totais de uma amostra e seus potenciais como antioxidantes, como expresso na Tabela 4.

As observações de que as amostras mais pigmentadas e/ou integrais apresentam maiores valores para componentes fenólicos eram esperadas e coincidem com dados apresentados por outros pesquisadores. Capacidade antioxidante maior para os grãos vermelhos e pretos também foi observada por WALTER et al. (2013), e por GOFFMAN e BERGMAN (2004) utilizando o ensaio de DPPH. Já em estudo de SOMPONG (2011), uma amostra de arroz vermelho apresentou valor de compostos fenólicos maiores do que o preto. MIN, LUNG e CHEN (2012), observaram que os níveis-limite DPPH de farelo de arroz vermelho e roxo foram superiores aos de cor clara.

O arroz selvagem, com coloração negra no pericarpo, também apresentou alto potencial antioxidante, embora cerca de 2 a 3 vezes menor em relação às demais amostras com coloração, esta diferença pode ser justificada em parte por este não ser considerado arroz e sim uma gramínea aquática, do gênero Zizania, nativo da América do Norte (BERTAZZONI e DAMASCENO-JUNIOR, 2011).

A redução de compostos fenólicos em amostras de grãos polidos foi observada por WALTER et al. (2013). O arroz branco "in natura", que passa por um processo padrão de beneficiamento para a retirada da casca e polimento (brunido), acaba por ter reduzidos seus teores de fenólicos e outros 
nutrientes. JUN et al. (2012) também observou esta diferença, quando a amostra de arroz integral obteve valores maiores de fenólicos totais e capacidade antioxidante, do que a forma polida.

A parboilização é um processo hidrotérmico, no qual o arroz em casca é imerso em água potável, a uma temperatura acima de $58^{\circ} \mathrm{C}$, seguido de gelatinização parcial ou total do amido e secagem (EMBRAPA, 2005), que poderia aumentar os teores de nutrientes e demais compostos bioativos nos grãos. Neste trabalho, não foi possível verificar uma relação positiva entre as amostras parboilizadas e os parâmetros aqui analisados.

GOUFO e TRINDADE (2014) afirmaram haver variação significativa também nos níveis de compostos antioxidantes do arroz, considerando como os principais fatores responsáveis a composição genética das diferentes variedades de arroz, fatores pré-colheita, condições de armazenamento e métodos analíticos. Embora neste estudo um baixo número de amostras orgânicas tenham sido estudadas, os dados de composição de fenólicos e potencial antioxidante indicam que estes grãos podem ser superiores aos seus correspondentes cultivados de maneira convencional, possivelmente como resultado do diferencial da produção orgânica, baseada em sistemas naturais equilibrados e férteis, que colaboram para um metabolismo diferenciado do vegetal (EMBRAPA, 2005) Quando a produção orgânica foi somada à apresentação do grão em sua forma integral, houve um destacado melhoramento no aspecto de capacidade antioxidante e conteúdo de fenólicos (Tabelas 3 e 4).

A redução nos teores de fenólicos totais após o aquecimento foi observada por vários estudos (FARES, 2010; FINOCCHIARO et al., 2007; XU e CHANG, 2008) variando de acordo com as condições e método de cultivo, espécies e partes das plantas, técnica de cocção, devido a perdas para a água de cocção e principalmente pelas interações possíveis com outros componentes dos grãos. RAWELL et al. (2001) demonstram que proteínas e componentes fenólicos de vegetais podem interagir por interações de hidrogênio, hidrofóbicas ou iônicas, e mesmo por ligações covalentes, sendo estas aceleradas pelo aquecimento e resultando na redução da disponibilidade e solubilidade de ambos.

\section{CONCLUSÃO}

Embora o tipo de arroz mais consumido entre os brasileiros seja o arroz branco e polido, há disponível no mercado outros tipos que podem ser mais ricos em componentes bioativos como os compostos fenólicos. Neste trabalho observou-se que as amostras de arroz de genótipos pigmentados apresentam altos teores de fenólicos totais, incluindo os flavonoides, o que poderia explicar seus altos valores para atividade antioxidante. As amostras de grãos integrais e orgânicos também se destacaram. Estes dados indicam que a substituição do arroz branco por grãos integrais, e, especialmente por grãos pigmentados, pode ser benéfica, contribuindo para o aumento da ingestão de componentes antioxidantes. 


\section{AGRADECIMENTOS}

Os autores agradecem à FAPEMIG - Fundação de Amparo à Pesquisa de Minas Gerais, pela Bolsa de Iniciação Científica à Ludmila Gontijo Pacheco, à Prof ${ }^{a}$ Dr $^{a}$ Roseli Aparecida da Silva Gomes, pela disponibilização de recursos do Laboratório de Bioquímica e Biofísica/ ICB/ UFTM, e à Jaime de Nóbrega pela revisão dos textos em língua estrangeira.

\section{REFERÊNCIAS}

ANGELO, P.M.; JORGE, N. Compostos Fenólicos em Alimentos - Uma Breve Revisão. Revista do Instituto Adolfo Lutz, v. 66, p. 232-240, 2007.

BARATA, T.S. Caracterização do consumo de arroz no Brasil: um estudo na Região Metropolitana de Porto Alegre. [mestrado]. Porto Alegre: Universidade Federal do Rio Grande do Sul - CEPAN; 2005.

BERTAZZONI, E.C.; DAMASCENO-JUNIOR, G.A. Aspectos da biologia e fenologia de Oryza latifolia Desv. (Poaceae) no Pantanal sul-mato-grossense. Acta Botânica Brasilica, v. 5, n. 2, p. 476-486, 2011.

BOATENG, J.; VERGHESE, M.; WALKER, L.T.; OGUTU, S. Effect of processing on antioxidant contents in selected dry beans (Phaseolus spp. L.). LWT-Food Science and Technology, v. 41, n. 9, p. 1541-1547, 2008.

BRAND-WILLIAMS, W.; CUVELIER, M.E.; BERSET, C. Use of a free radical method to evaluate antioxidant activity. LWT-Food Science and Technology, v. 28, n. 1, p. 25-30, 1995.

CANO, A.; ACOSTA, M.; ARNAO, M. Hydrophilic and lipophilic antioxidant activities changes during on vine ripening of tomatoes (Lycopersicon esculentum Mill.). Postharvest Biological Technology, v. 28, n. 1, p. 59-65, 2003.

CHO, J.G.; SONG, N.Y.; NAM, T.G.; SHRESTHA, S.; PARK, H.J.; LYU, H.N.; KIM, D.O.; LEE, G.; WOO, Y.M.; JEONG, T.S.; BAEK, N.I. Flavonoids from the grains of C1/R-S transgenic rice, the transgenic Oryza sativa spp. japonica, and their radical scavenging activities. Journal of Agricultural and Food Chemistry, v. 61, n. 43, p. 10354-10359, 2013.

DEMMING-ADAMS, B.; ADAMS, W.W. Antioxidants in Photosynthesis and Human Nutrition. Science, v. 298, p. 2149-2153, 2002. 
EMPRESA BRASILEIRA DE PESQUISA AGROPECUÁRIA - Embrapa Clima Temperado. Consumo, Mercado e Comercialização do Arroz no Brasil - Arroz Irrigado: recomendações técnicas da pesquisa para o Sul do Brasil, 2005 [Internet]. [cited 2014 mar 20] Pelotas: Embrapa Clima Temperado/IRGA/ EPAGRI. Available from: https://bit.ly/3c8EgMc.

FARES, C.; PLATANIA, C.; BAIANO, A.; MENGA, V. Effect of processing and cooking on phenolic acid profile and antioxidant capacity of durum wheat pasta enriched with debranning fractions of wheat. Food Chemistry, v. 119, p. 1023-1029, 2010.

FINOCCHIARO, F.; FERRARI, B.; GIANINETTI, A.; DALL'ASTA, C.; GALAVERNA, G.; SCAZZINA, F.; PELEGRINNI, N. Characterization of antioxidant compounds of red and white rice and changes in total antioxidant capacity during processing. Molecular Nutrition Food Research, v. 51, p. 1006-1019, 2007.

FOOD AND AGRICULTURE ORGANIZATION OF THE UNITED NATIONS - FAOSTAT. Food Balance Sheets. 2011 [Internet]. [cited 2019 dez 29]. Available from: https://bit.ly/2zf0rC0.

HOROWITZ, W. (Ed.). Official Methods of Analysis of the Association of Official Analytical Chemists. 17th ed. Washington: AOAC, 2000. 2200 p.

GOFFMAN, F.D.; BERGMAN, C.J. Rice kernel phenolic content and its relationship with antiradical efficiency. Journal of Science and Food Agricultural, 84, n. 10, p. 1235-1240, 2004.

GOUFO, P.; TRINDADE, H. Rice antioxidants: phenolic acids, flavonoids, anthocyanins, proanthocyanidins, tocopherols, tocotrienols, $\gamma$-oryzanol, and phytic acid. Food Science and Nutrition, v. 2 , n. 2, p. 75-104, 2014.

HUDSON, E.A.; DINH, P.A.; KOKUBUN, T.; SIMMONDS, M.S.J.; GESCHER, A. Characterization of potentially chemopreventive phenols in extracts of brown rice that inhibit the growth of human breast and colon cancer cells. Cancer Epidemiology, Biomarkers \& Prevention, v. 9, p.1163-1170, 2000.

INSTITUTO BRASILEIRO DE GEOGRAFIA E ESTATÍSTICA - IBGE. Pesquisa de Orçamentos Familiares 2008-2009. Análise do Consumo Alimentar Pessoal no Brasil. Rio de Janeiro: IBGE; 2010.

JUN, H.I.; SONG, G.S.; YANG, E.I.; YOUN, Y.; KIM, Y.S. Antioxidant activities and phenolic compounds of pigmented rice bran extracts. Journal of Food Science, v. 77, n. 7, p. C759-764, 2012. 
MIN, B.; M.C.C.; LUNG, A.M.; CHEN, M.H. Phytochemicals and Antioxidant Capacities in Rice Brans of Different Color. Journal of Food Sciece, v. 76, n. 1, p. C117-C126, 2011.

NAVES, M.M.V. Características químicas e nutricionais do arroz. Boletim do CEPPA, v. 25, p. 51-60, 2007.

NIKI, E. Assessment of Antioxidant Capacity in vitro and in vivo. Free Radical Biology \& Medicine, v. 49, p. 503-515, 2010.

PEREIRA, A.L.F; VIDAL, T.F.; CONSTANT, P.B.L. Dietary antioxidants: chemical and biological importance. Nutrire, v. 34, n. 3, p. 231-247, 2009.

PEREIRA, M.P.; TAVANO, O.L. Use of different spices as potential natural antioxidant additives on cooked beans (Phaseolus vulgaris). Increase of DPPH radical scavenging activity and total phenolic content. Plant Foods for Human Nutrition, v. 69, n. 4, p. 337-343, 2014.

RAWEL, H.M.; KROLL, J.; HOHL, U.C. Model studies on reactions of plant phenols with whey proteins. Nahrung, v. 45, n. 2, p. 72-81, 2001.

SILVA, M.L.C.; COSTA, R.S.; SANTANA, A.S.; KOBLITZ, M.G.B.; Compostos fenólicos, carotenóides e atividade antioxidante em produtos vegetais. Semina: Ciências Agrárias, v. 31, n. 3, p. 669-682, 2010.

SOMPONG, R.; SIEBENHANDL-EHN, S.; LINSBERGER-MARTIN, G.; BERGHOFER, E. Physicochemical and antioxidative properties of red and black rice varieties from Thailand, China and Sri Lanka. Food Chemistry, v. 124, p. 132-140, 2011.

SOOBRATTEE, M. A.; NEERGHENN, V. S.; LUXIMON-RAMMA, A.; ARUOMA, O. I.; BAHORUN, T. Phenolics as potential antioxidant therapeutic agents: Mechanism and actions. Mutation Research, v. 579, p. 200-213, 2005.

STATISTICA 8.0. Statistics computer program for Windows, v.1. Tulsa: StatSoft Inc. Software, 2007.

THOMAS, R.H.; BERNARDS, M.A.; DRAKE, E.E.; GUGLIELMO, C.G. Changes in the antioxidant activities of seven herb- and spice-based marinating sauces after cooking. Journal of Food Composition and Analysis, v. 252, n. 23, p. 244-252, 2010. 
VICHAPONG, J.; SOOKSERM, M.; SRIJESDARUK, V.; SWATSITANG, P.; SRIJARANAI, S. High performance liquid chromatographic analysis of phenolic compounds and their antioxidant activities in rice varieties. Food Science and Technology, v. 43, p. 1325-1330, 2010.

WALTER, M.; MARCHESAN, E.; MASSONI, P.F.S.; SILVA, L.P.; SARTORI, G.M.S; FERREIRA, R.B. Antioxidant properties of rice grains with light brown, red and black pericarp colors and the effect of processing. Food Research International, v. 50, p. 698-703, 2013.

XU, B.J.; CHANG, S.K.C. Total Phenolic Content and Antioxidant Properties of Eclipse Black Beans (Phaseolus vulgaris L.) as Affected by Processing Methods. Journal of Food Science, v. 73, n. 2, p. 19-27, 2008.

ZIMMERMANN, A.M.; KIRSTEN, V.R. Alimentos com função antioxidante em doenças crônicas: uma abordagem clínica. Ciências da Saúde, v. 9, n. 1, p. 51-68, 2008. 